Published in Swiss Medical Informatics (SMI), 2015, vol. 32, p. 1-7 which should be cited to refer to this work.

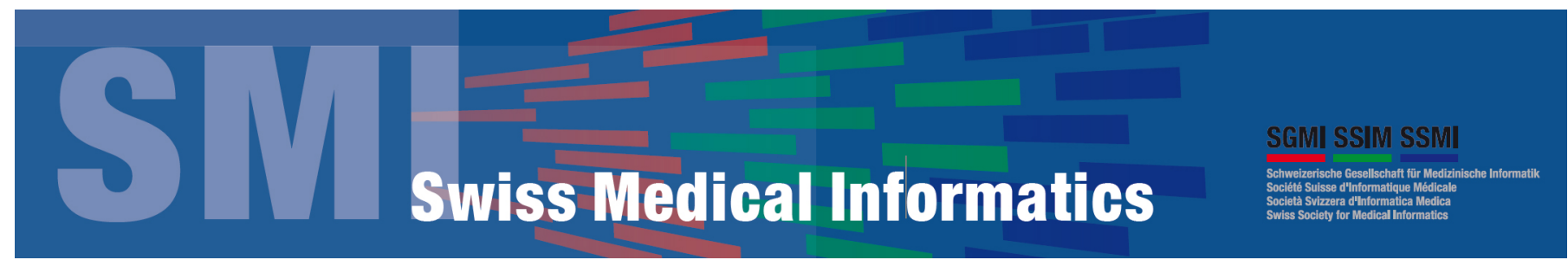

\title{
DiagnosticAid: Une plateforme collaborative pour les guides de pratique en médecine générale
}

\author{
Bruno Alves ${ }^{a}$, Michael Ignaz Schumacher ${ }^{a}$, Samuel Gaillard ${ }^{b}$, Johannes Krampf ${ }^{a}$, Fabian Imsand ${ }^{a}$ \\ ${ }^{a}$ Institut Informatique de Gestion, HES-SO, Sierre \\ ${ }^{\mathrm{b}}$ Logival SA, Sierre
}

\section{Summary}

DiagnosticAid: A collaborative platform for clinical guidelines adapted to the general practice

A doctor's job consists in making decisions in an environment of uncertainty. Certain tools and aids can help in this decision-making process, such as medical practice guidelines. When computerized, these save time and reduce error. The DiagnosticAid project aims to provide doctors with a computerized help tool for effective decision making based on medical practice guidelines developed for general medicine. Our in-depth knowledge of consulting processes has enabled us to develop a specialized tool as well as implement specially-designed test scenarios. In order to develop it, various new technologies had to be created, including a runtime environment for practice guidelines. To this end, we have developed a model, serialization, formula-based language, and runtime engine. This paper outlines in four sections the development of the different technologies.

\section{Introduction}

Le travail du médecin généraliste consiste souvent à faire face à l'incertitude. Il est régulièrement confronté à de multiples hypothèses, qu'il cherche à étayer par des observations, ses connaissances ou son expérience. Parfois, cette confrontation peut générer un stress, rendre la consultation inefficace ou une trop grande utilisation de ressources [1]. Dans ces cas limite, sa connaissance ne suffit plus et il doit alors s'en remettre à des procédures écrites basées sur la pratique ou sur l'évidence médicale: les guides de pratique. Plusieurs études $[2,3]$ ont déjà montré que l'utilisation de guides de pratique informatisés permet un gain de temps en consultation, une amélioration de la qualité, ainsi qu'une diminution significative des erreurs de diagnostic. De plus, la connaissance transcrite dans de tels guides est bien supérieure à celle du médecin seul.

Les médecins romands utilisent depuis longtemps le recueil de guides «Docteur, J'ai...» [4], co-écrit par le Dr. MarcAndré Raetzo. En cas de doute, le généraliste consulte ce livre pour se rassurer sur la marche à suivre. Toutefois, bien que cet ouvrage soit très pratique dans la plupart des cas, son utilisation génère des contretemps et n'est plus adaptée à une pratique basée sur les outils informatiques.
DiagnosticAid est une collaboration entre Logival SA et l'Institut Informatique de gestion de la HES-SO financée par le CTI/KTI. Le but du projet est de fournir aux cabinets médicaux privés un système d'aide au diagnostic basé sur les guides de pratique clinique informatisés. Le système permet de créer, modifier, exécuter et partager ces outils. Il vise à faciliter le travail des praticiens pour mieux gérer leur temps de consultation et réduire le nombre d'erreurs de diagnostic. Pour qu'un tel système soit possible, des solutions d'interopérabilité ont été mises en place pour récupérer une quantité suffisante de données.

Ce projet s'appuie notamment sur un environnement de gestion et d'exécution de guides informatisés ciblés sur la médecine générale. Cependant, le cadre du projet est bien plus large, avec le développement de passerelles vers les réseaux de soins (Infomed et Vivates), l'implémentation de la spécification SMEEX, etc. Toutefois, nous limiterons la discussion à la partie du projet en lien avec les guides informatisés.

Cet article est structuré en 4 parties. La section suivante introduit la motivation d'un tel projet, ainsi que la méthodologie utilisée. La partie suivante détaille les composants essentiels de la technologie. Une courte discussion en fin de papier renseigne sur les points manquants pour finir sur la conclusion du travail.

\section{Méthodologie}

\section{De l'hypothèse au diagnostic}

Mediway, le dossier patient informatisé (DPI) leader du marché Suisse Romand a été développé par Logival SA. Le logiciel possède une implémentation rudimentaire des guides informatisés. Le recueil «Docteur, j'ai...» est notamment digitalisé sous forme de formulaires à score. DiagnosticAid cherche ainsi à améliorer cette intégration à en proposant un ensemble cohérent de technologies de support à la décision. Ce support se fait sous la forme d'un système de gestion de guides de pratique informatisés.

A l'inverse des guides de pratique clinique, les guides informatisés pour médecine générale ne mettent pas l'accent sur le diagnostic, puisque celui-ci est inconnu. Ces guides mènent le praticien étape par étape vers une confirmation / un rejet d'une hypothèse de départ. Le processus s'arrête lorsque la probabilité est suffisante pour considérer le diagnostic comme fiable. 


\section{Processus de consultation}

Le projet a débuté par une enquête auprès d'un panel de médecins utilisant le logiciel Mediway. Le but de celle-ci était de se familiariser avec le processus de consultation et sur les habitudes des différents médecins. Ce processus de déroule ainsi:

a. Le médecin pose des questions générales au patient sur son état actuel;

b. Le médecin formule des hypothèses sur le problème à traiter, qu'il vérifie par des questions toujours plus précises;

c. Le médecin effectue des examens, tests et prélèvements pour vérifier ces hypothèses;

d. Le processus s'arrête dès que le seuil de probabilité fixé arbitrairement pour le médecin est dépassé;

e. La cause est alors suffisamment «certaine» pour être ciblée par un traitement adéquat.

Dans son investigation, le médecin peut s'appuyer sur les outils suivants:

- Questions: le praticien pose les bonnes questions pour cibler le problème sur la base de ses hypothèses et observations;

- Actions: le praticien effectue des examens et des prises d'échantillons qui permettent de raffiner la recherche des causes;

- Décisions: le praticien prend des décisions éclairées basé sur les probabilités d'une cause (prescription par exemple)

Le projet DiagnosticAid cherche à transcrire ce savoir sous forme électronique, afin de fournir au médecin un outil de support à la décision efficace et discret. Afin de tester les outils, nous avons mis en place deux scénarios avec le Dr. Marc-André Raetzo: le calcul de risque cardio-vasculaire (Figure 1) et la suspicion d'embolie pulmonaire (Figure 2). Ces scénarios ont été sélectionnés, car ils imposent à peu près toutes les possibilités au moteur d'exécution.

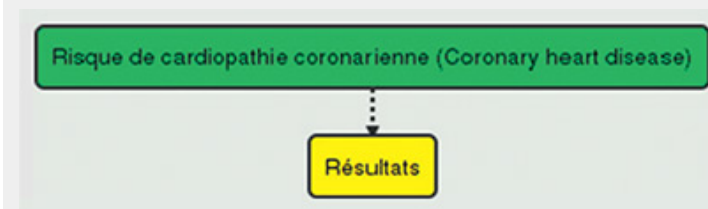

Figure 1

Calcul du risque cardio-vasculaire.

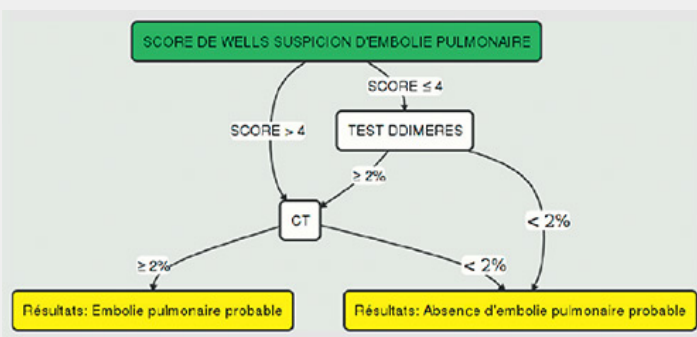

Figure 2

Suspicion d'embolie pulmonaire.

\section{Guides de pratique informatisés}

Dans le domaine du traitement de l'information, un guide interprétable par ordinateur (GIO), ou guide informatisé (GI) repose sur trois composants essentiels: le modèle, un langage d'expression et un moteur d'exécution.

\section{Modèle de guide}

Le modèle est un formalisme permettant de décrire un guide de pratique par ses composants et les relations entre eux. A cet égard, un guide de pratique n'est ni plus ni moins qu'un arbre de décision.

Pour transcrire une situation clinique dans ce modèle, il faut d'abord identifier clairement les étapes du processus (les nœuds), les seuils de déclenchement, l'hypothèse de départ (ainsi que sa probabilité) et aussi les résultats possibles. Chaque nœud définit un ensemble de questions à poser et chaque question définit à son tour une variable de modèle, utilisée pour représenter la réponse du patient à cette même question.

\section{Langage d'expression}

Afin de définir les états suivants dans l'arbre de décision, il est nécessaire de calculer des actions sur la base des questions posées. Pour ce faire, le moteur d'exécution doit pouvoir exécuter des formules arbitrairement complexes qu'il couple aux variables de modèle. Cet outil permet donc de paramétrer les conditions de déclenchement d'une action.

\section{Moteur d'exécution}

Le moteur d'exécution utilise les deux composants précédents pour exécuter un guide de pratique. Exécuter dans ce contexte veut dire parcourir l'arbre de la racine jusqu'à une de ces feuilles sur la base des réponses données et des seuils de déclenchement. Un guide est terminé, lorsque son état actuel est une décision. Dans les systèmes plus complexes, il arrive qu'un nœud de l'arbre puisse être lui-même une référence vers un autre guide. Nous avons choisi dans DiagnosticAid d'écarter cette possibilité.

\section{Analyse}

Nous avons effectué une analyse d'un certain nombre d'environnements d'exécutions de guides, tels que décrits dans $[5,6]$. Cette analyse s'est portée sur ASBRU, PROFORMA, GLIF3, EON et PRODIGY. Nous les avons analysés sur trois axes, à savoir: expressivité, simplicité et outils disponibles. Nous avons conclu, que la difficulté à intégrer ces outils dans notre environnement de travail était un frein à leur utilisation. De plus, nous avons considéré que l'avantage business d'un développement propre était grand, car maîtriser notre propre environnement, nous permettait de contrôler son évolution.

\section{Résultats}

Un ensemble de technologies ont été développées:

- Un environnement d'exécution de guides

- Un portail en ligne de gestion et de partage de guides

\section{Environnement d'exécution}

L'environnement d'exécution a été développé en PHP. Contrairement aux «moteurs de workflow» classiques, qui 
cherchent à automatiser des activités, notre moteur cherche à calculer la probabilité d'une hypothèse à la sortie d'un nœud.

\section{Guideline Modelling Language (GML)}

Le modèle de guides s'appuie sur les primitives suivantes:

- Nœud de calcul

- Nœud de score

- Nœud de Bayes

- Nœud décisionnel

Nous avons créé pour ce modèle une sérialisation en XML. Noud de calcul: Le nœud de calcul permet de calculer une condition de déclenchement sur la base d'un calcul complexe faisant intervenir les variables de modèle. La valeur obtenue est comparée à un seuil de déclenchement pour décider de l'action à entreprendre. La probabilité des actions peut être fixée par l'utilisateur ou dépendre d'une formule. La probabilité à posteriori est calculée lors du déclenchement de l'action et dépend de la probabilité à priori du nœud.

Noud de score: Le nœud de score est une spécialisation du nœud de calcul. Le résultat est un score obtenu en additionnant les valeurs des réponses données par le patient aux questions posées. Les conditions de déclenchement de l'action et le calcul de probabilités sont les mêmes que pour le nœud de calcul.

Noud de Bayes: Le nœud de Bayes calcule les valeurs prédictives d'un test (à postériori) en utilisant la prévalence de celui-ci (probabilité à priori), la sensibilité et la spécificité données par le couple médecin-patient:

$$
\begin{aligned}
& \text { PPV }=\frac{\text { sens } \cdot \text { prev }}{\text { sens } \cdot \text { prev }+(1-\text { spec }) \cdot(1-\text { prev })} \\
& \text { NPV }=\frac{\text { spec } \cdot(1-\text { prev })}{(1-\text { sens }) \cdot \operatorname{prev}+\text { spec } \cdot(1-\text { prev })}
\end{aligned}
$$

Un nœud de Bayes est un test avec une question de type oui/non. La valeur prédictive calculée est soit positive (PPV), soit négative (NPV).

Noud décisionnel: Le nœud décisionnel est une feuille de l'arbre décisionnel. Il est constitué d'un ensemble de décisions. Pour chaque décision, le nœud contient une liste de conséquences qui peuvent être des effets bénéfiques, secondaires ou secondaires dépendant d'une probabilité. Pour ce dernier cas, probabilités sont calculées, ainsi que les valeurs NNT et NNH.

\section{Guideline Expression Language (GEL)}

Le langage d'expression GEL fournit une syntaxe proche de celle de Microsoft Excel 2007. Il s'agit d'un langage de formules qui utilise environ $70 \%$ des constructions et fonctions de son modèle. Ce choix a été motivé par le fait que beaucoup de médecins sont déjà bien entraînés au langage MS-Excel.

Le GEL supporte les opérateurs logiques (ET, OU, NON), de comparaison $(<,<=,=,<>,>=,>)$, arithmétiques $(+,-$, $*, /, \%, \wedge)$, unaires $(\sim, !,++,--)$, les variables et les fonctions. Il supporte, tout comme son modèle, les fonctions à paramètres variables (ex: OR(true; true; ...; false))
Les fonctions fournies de base couvrent les domaines:

- Branchement (IF, SWITCH)

- Logique (AND, OR, NOT, XOR)

- Arithmétique (ABS, POW, ...)

- Trigonométrique (COS, ACOS, ...)

- Combinatoire (COMBIN, FACT, ...)

- Conversion (RAD, DEG)

Les calculs de déclenchement formulés à l'aide de ce langage sont contextualisés en donnant aux variables les valeurs obtenues par les réponses aux questions. Par exemple:

$$
\begin{aligned}
& \text { W } \leftarrow 28.4441 \text { * FEMALE }-1.4792 \text { * LN (AGE) - } \\
& 14.4588 \text { * FEMALE * LN (SYSTOLIC) }
\end{aligned}
$$

Ici, les variables FEMALE, AGE et SYSTOLIC proviennent de la réponse à 3 questions.

\section{Guideline Execution Engine (GEE)}

Le moteur d'exécution est de type «diagramme de flux». Il est chargé de calculer un chemin dans l'arbre décisionnel. A chaque itération, le GEE transcrit les valeurs des variables, effectue les calculs associé au nœud et avance dans l'arbre selon l'action programmée.

Les itérations continuent jusqu'à ce que le moteur rencontre sur un nœud décisionnel. A ce stade, les probabilités des conséquences liées aux décisions du nœud sont calculées et le moteur termine la session.

\section{Portail en ligne}

\section{Interface}

Le portail s'appuie sur les technologies développées afin de fournir une plateforme de création et de partage de guides. Chaque médecin peut créer ses propres guides, les éditer et les partager de façon simple et graphique. Toutefois, les guides ne peuvent être partagés avec la communauté qu'après avoir été contrôlés et vérifiés par des experts, euxmêmes utilisateurs de la plateforme.

\section{Application Programming Interface (API)}

La plateforme dispose d'une API permettant à ces solutions externes (notamment Mediway) de s'interfacer afin de récupérer automatiquement les résultats dans le dossier patient.

\section{Discussion}

Afin de simplifier le développement, nous avons décidé de créer notre propre environnement d'exécution de guides de pratique. Bien que ce choix soit justifiable aussi bien $\mathrm{du}$ point de vue technique, que business, la conséquence directe est que nous ne supportons qu'une partie des constructions supportées pas les autres environnements. Il nous manque notamment les patterns temporels et le parallélisme. Cela laissera donc une marge de manœuvre pour de futurs développements.

Ensuite, le grand désavantage d'une plateforme comme celle développée dans le cadre de ce projet est qu'il faut partir d'une hypothèse. Cela implique que le médecin puisse facilement trouver un guide qui corresponde à un en- 
semble de symptômes liés au problème. Or, cela n'est pour l'instant pas possible. En effet, le filtrage des guides se fait uniquement par catégorie ou par nom. Une future évolution devra donc corriger cela.

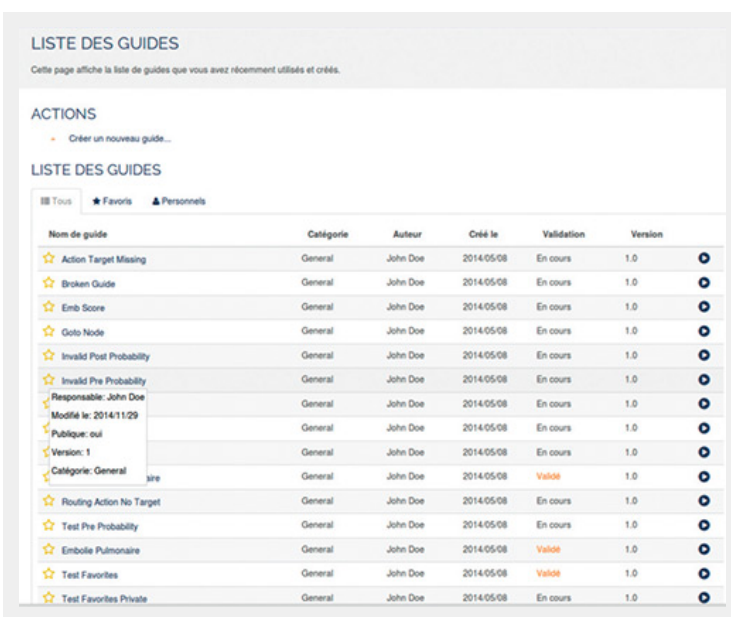

\section{Figure 3}

Vue du dashboard.

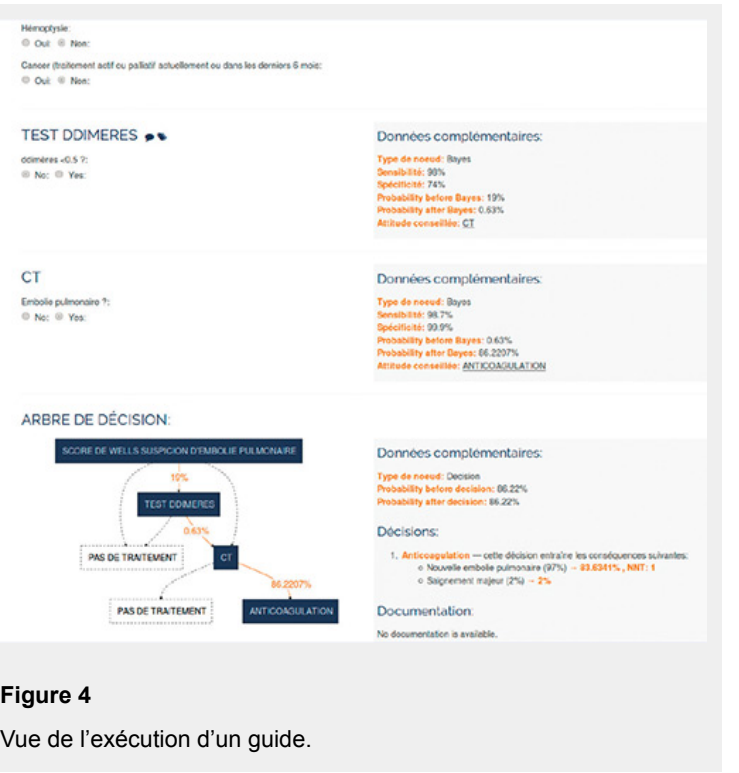

Des use-cases réalistes ont été choisis pour tester la plateforme. Toutefois, il est indispensable pour nous d'amorcer la commercialisation de cette plateforme avec un nombre conséquent de guides pré-digitalisés. C'est pourquoi nous allons faire appel à des experts pour la transcription des guides du recueil «Docteur, j'ai...». Le Dr. Marc-André Raetzo lui-même supervisera leur création.

\section{Conclusion}

En conclusion, le projet DiagnosticAid a permis de mettre en place une plateforme de création et de partage de guides de pratique pour la médecine générale. La plateforme est actuellement en cours d'amélioration en vue de sa commercialisation. Les retours des testeurs sont très bons et les médecins sont positifs à l'utilisation d'une telle extension de leur environnement de travail.

\section{Correspondance:}

Bruno Alves

Institut Informatique de Gestion

HES-SO Valais-Wallis

Rte de la Plaine 2

Case postale 80

$\mathrm{CH}$-3960 Sierre

bruno.alves[at]hevs.ch

\section{Références}

1 Gerrity MS, DeVellis RF, Earp JA. Physicians' reactions to uncertainty in patient care: a new measure and new insights. Med Care. 1990;724-36.

2 Grimshaw JM, Russell IT. Effect of clinical guidelines on medical practice: A systematic review of rigorous evaluations. Obstet Gynecol Survey. 1993;49(7):469-70.

3 Woolf SH, Grol R, Hutchinson A, Eccles M, Grimshaw J. Clinical guidelines: potential benefits, limitations, and harms of clinical guidelines. BMJ. 1999;318(7182):527.

4 Raetzo M-A, Restellini A. Docteur, j'ai ...: stratégies diagnostiques et thérapeutiques en médecine ambulatoire. Médecine et Hygiène. 1996.

5 Peleg M. Computer-interpretable clinical guidelines: A methodological review. J Biomed Informatics. 2013;46(4):744-63.

6 Peleg M, Tu S, Bury J, Ciccarese P, Fox J, et al. Comparing computerinterpretable guideline models: a case-study approach. J Am Med Informat Ass. 2003;10(1):52-68. 
Risque de cardiopathie coronarienne (Coronary heart disease)

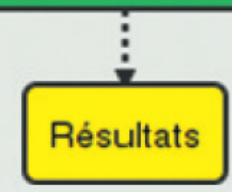

Figure 1

Calcul du risque cardio-vasculaire.

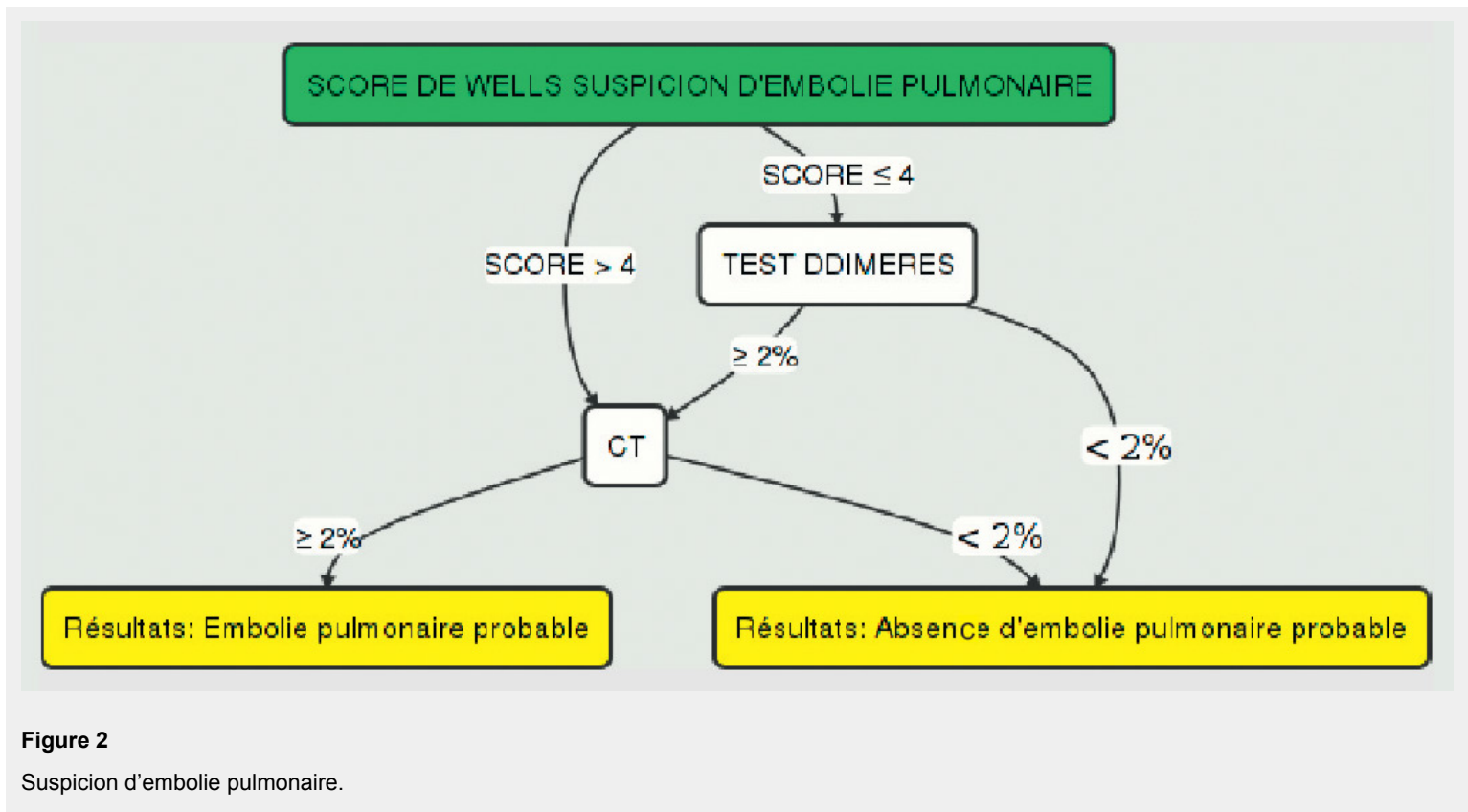




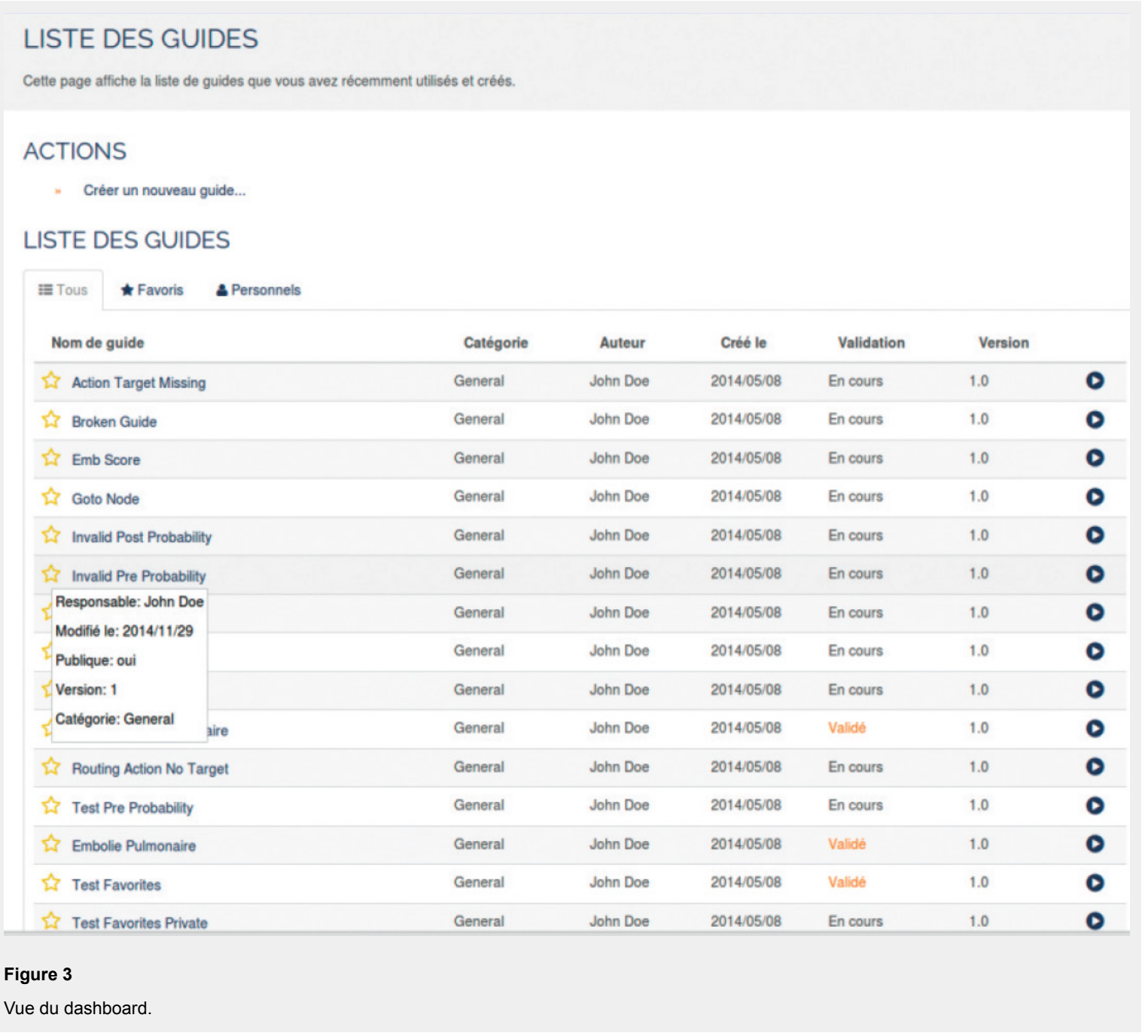




\section{Original article}

Hémoptysie:

Oui: Non:

Cancer (traitement actif ou palliatif actuellement ou dans les derniers 6 mois:

Oui: Non:

\section{TEST DDIMERES}

ddimères $<0.5$ ?

(1) No: Yes:

\section{CT}

Embolie pulmonaire ?:

No: $\odot$ Yes:

\section{Données complementaires:}

Type de noeud: Bayes

Sensibilité: $98 \%$

Spéciticité: $74 \%$

Probability before Bayes: $19 \%$

Probability after Bayes: $0.63 \%$

Attitude conseillée: $\underline{\mathrm{CT}}$

\section{Données complémentaires}

Type de noeud: Bayes

Sensibilité: $98.7 \%$

Spécificité: $99.9 \%$

Probability before Bayes: $0.63 \%$

Probability after Bayes: $86.2207 \%$

Attitude conseillée: ANTICOAGULATION

\section{Donnees complementaires:}

Type de noeud: Decision

Probability before decision: $86.22 \%$

Probability after decision: $\mathbf{8 6 . 2 2 \%}$

\section{Décisions:}

1. Anticoagulation - cette décision entraîne les conséquences suivantes: - Nouvelle embolie pulmonaire $(97 \%) \rightarrow 83.6341 \%$, NNT: 1 - Saignement majeur $(2 \%) \rightarrow 2 \%$

Documentation:

No documentation is available.

Figure 4

Vue de l'exécution d'un guide. 